Informatics \& Technology | Alexander Scheinker

\title{
Optimising Particle Accelerators with Adaptive Machine Learning
}

Machine learning has become a staple of research into many of today's most cutting-edge technologies. Until now, however, it has not been
widely considered as a useful widely considered as a useful
tool for online optimisation of the performance of particle the performance of particle accelerators. Through his Scheinker at the Los Alamos National Laboratory in New Mexico, USA, introduces new techniques based on machine learning, which can adaptively tune the bewilderingly complex controls of particle accelerators in real the without human intimately make it far could for researchers across many different academic fields to make ground-breaking new discoveries.

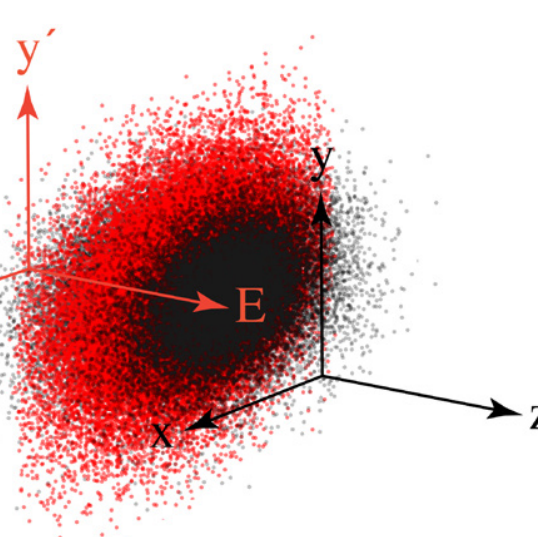

Particle dynamics live in $6 \mathrm{D}$ space. Particle
locations $(x, y)$ are shoun in black locations $(x, y, z)$ are shown in black and the ang
at which they are flying relative to the $z$-axis an
their energies
$x^{\prime} y$ oday's particle accelerators are some of the greatest feats of human engineering ever achieved, enabling researchers to answer fundamental questions in fields ranging from medicine to cosmology. Which such a diverse and complex array accelerator failities, different research groups may require radically different characteristics in the particle beams they produce. To accommodate such variation, every last intricate compon of a particle accelerator must be adjustable, without sacrificing the overal performance of the device.

"Accelerators are kilometre-scale machines with thousands of coupled components that accelerate, turn, and focus their beams", Dr Alexander Scheinker, researcher at the Los Alamos National Laboratory in New Mexico, USA, describes. "Beams must be precisely controlled to achieve desired pream energlos and lengths, and to time." Traditionally, achieving these optimised conditions has required researchers and engineers to spend many hours on manual tuning. With many research groups jostling for use of the apparatus, this eats into valuable time which could be spent gathering data, ultimately making it far more .

\section{THE POWER OF}

\section{MACHINE LEARNIN}

To overcome this problem, Dr Scheinke proposes that the role of particle accelerator tuning can be aided by Where machine learning algorithms. minds a limited in ther ablina simultaneously take into account the role of every tuneable component (out of thousands) in a particle accelerator, these artificial intelligence algorithm build up their own models of highly intricate systems based on real data. After being trained in this way, almost instantaneously make the own predictions of how these systems will behave in certain situations. More specifically, 'neural networks' are machine learning algorithms which can mimic the decisions made by human brains. They can be trained using information detailing the varying outcomes of different tasks as they are performed on a system.

Crucially, neural networks then learn by hemselves how a certain task should be carried out in order to produce the best possible outcome, without being programmed beforehand. "Machine learning tools such as neural networks have recently grown in popularity from identifying cats to developing antibiotics", explains Dr Scheinker. "Neural networks are useful because of their ability to learn input-output relationships of complex systems." Dr Scheinker believes that these tools provide researchers with ideal solutions to the problems posed by particle accelerator control. All the same, implementing them for use in significant challenges.

\section{ACCOUNTING FOR}

\section{WANDERING PARTICLES}

The underlying challenge posed by unwanted yt in in the motions of its particles. Ideally. they would all closely follow a central axis oriented along the direction of and would have relatively uniform energies. If not controlled properly however, particles will also travel in both perpendicular directions to the axis, fly off at different angles, and adopt more widely spread energies. Together, these distortions will quickly build up over time, and severely limit the quality of the beam.

These problematic movements are exacerbated by the convoluted webs of interactions between individual particles, which are all but impossible to map out using conventional machine learning approaches and uncertain and As Dr Scheinker desc components. As Dr Scheinker describes, "one of the based model such as a neural network to the real time active control of a particle accelerator is the fact that both the accelerator components and the beams themselves drift and change unpredictably with time. This means that the predictive power of any model based on a set of data will degrade quickly." With no clear way of knowing how these unwanted motions will play out, researchers must find innovative ways to deal with them while the equipment is running, without relying on models buit around previously gathered data.

\section{REAL-TIME TUNING}

As part of his $\mathrm{PhD}$, Dr Scheinker and his to devise a modelin Dr Scheinker's technique has been

implemented for a device named a free electron laser (FEL) - in which groups of electrons travel at close to the speed of ght through a specialised arrangement of magnets. These devices are highly tuneable and create brighter and more coherent flashes of light, by many orders of magnitude, than any other type of laser, making them incredibly useful for material science and biology studies. However, because of the random nature of the FEL process and the many interactions that take place within groups of such rapidy moving electrons, the detalled particle distributions are

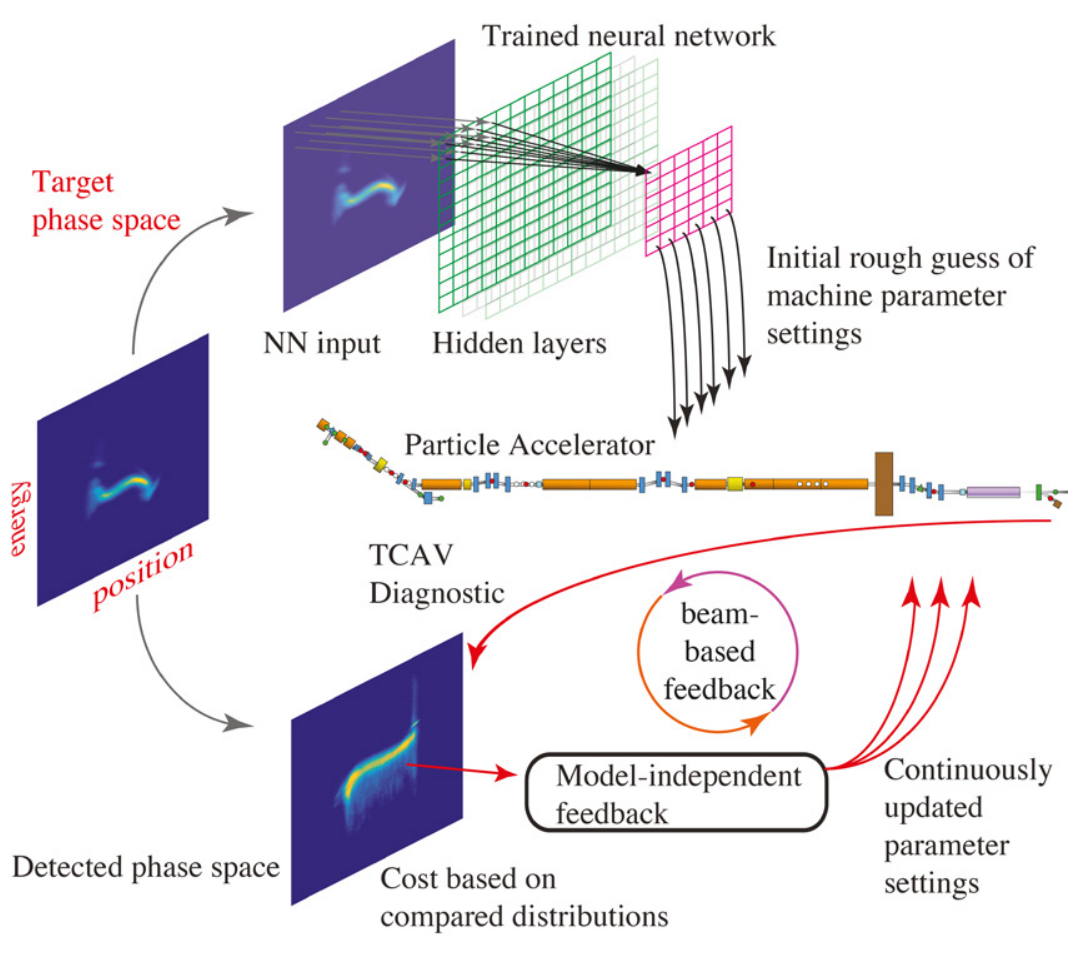

Dr Scheinker proposes that the role of particle accelerator tuning can be aided by adaptive machine learning algorithms.

that FELs are inherently noisy even when their tuning parameters are kept constant - degrading the repeatability and accuracy of experimental outcom and making tuning and optimisation using traditional approaches difficult. Dr Scheinker's team combated this model-independent 'feedback contro' system, which used the noisy data FEL light to deternin hories of be adjusted to maintain its desirable properties. This allowed up to 105 components to be tuned simultaneouly from real-time data, independent of any data-based model.

As Dr Scheinker summarises, "we have demonstrated this method for maximising the output energy of the light produced from an FEL by tuning arous and for ad insy energy measurement and for adaptively tuning online models to automatically track time-varying create non Yet despite the clear success of this technique, it still faced further problems. team's feedback control system could

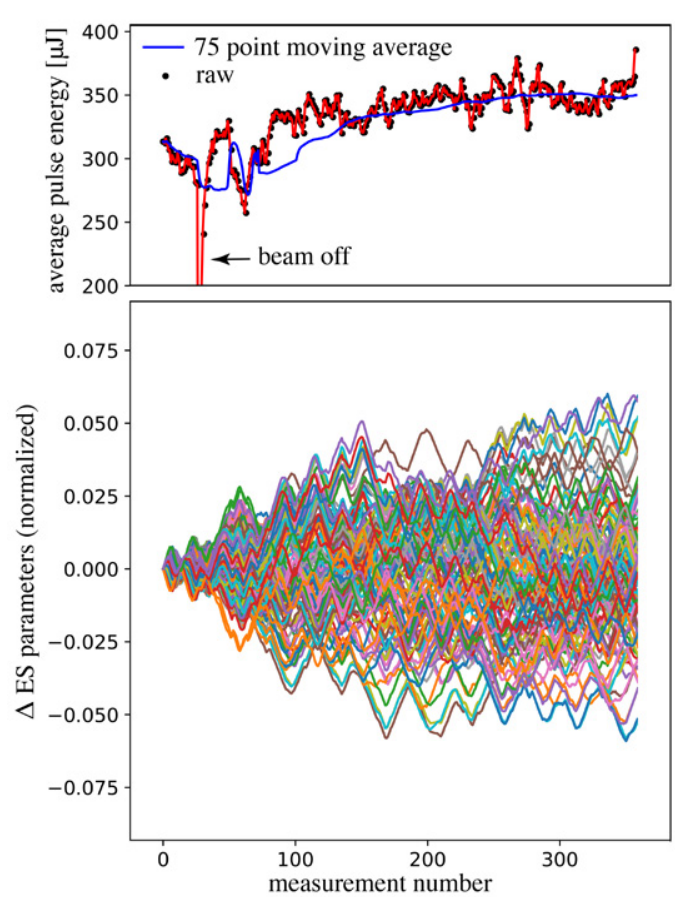

Experimental results of tuning around 100 magnets simultaneously to maximise the noisy output 

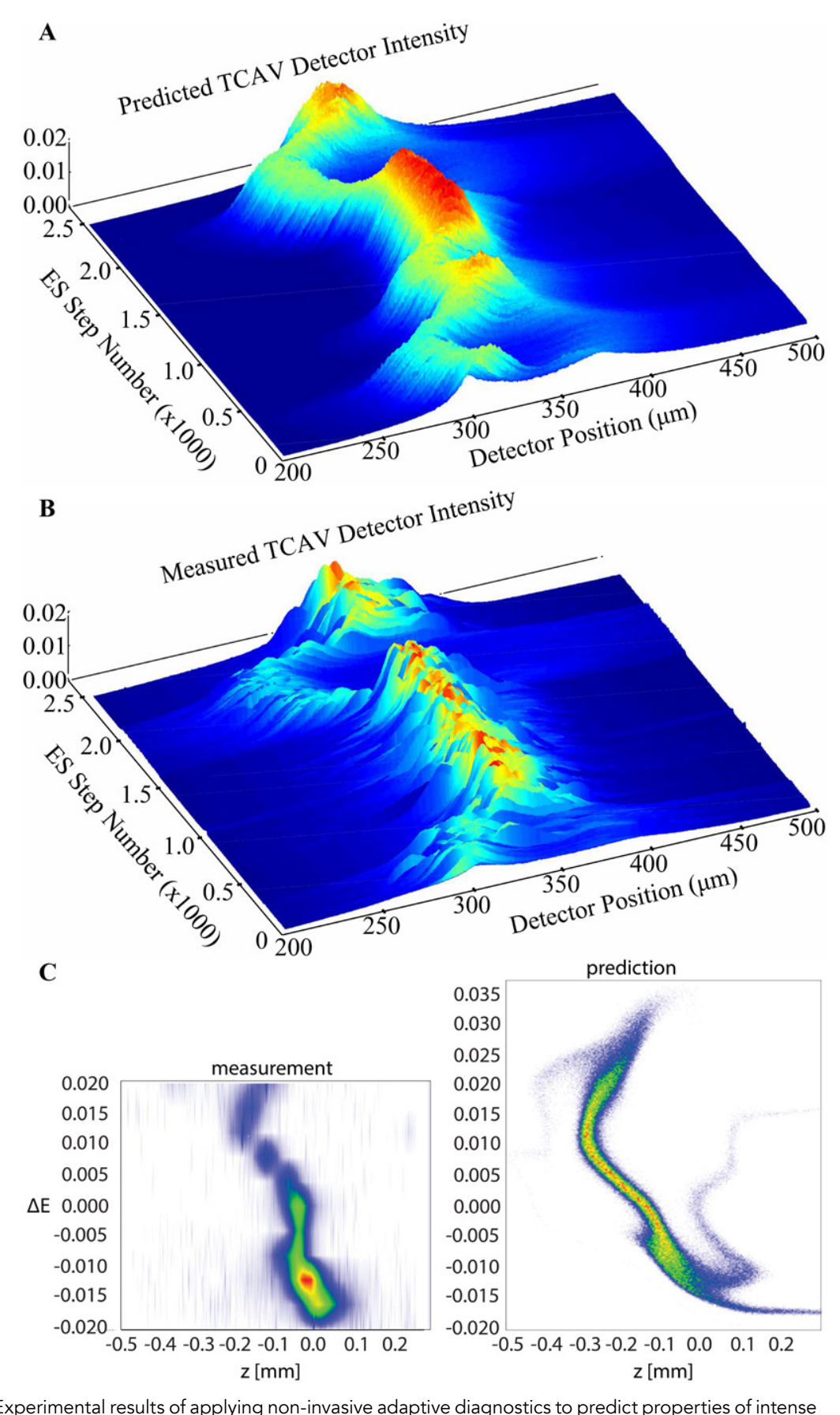

Experimental results of applying non-invasive adaptive diagnostics to predict properties of intense

This active feedlback control approach could be applied universally to any complex system which varies unpredictably over time.

become 'stuck' in one solution which was reasonably good, but which didn't

CONSIDERING EVERY PARAMETER their latest research, Dr Scheinker and machine learning feedback control approach for noisy and time-varying systems. This approach combined the fobustness of model-independent feedback with the global learning previous studies hat aready. As he algorithms are well suited to (eaning about every aspect of unknown over time. They demonstrated this innovative approach using the Linac Coherent Light Source (LCLS) at Stanford University - which explots the high tunability of FELs to produce high-quality X-ray pulses lasting just quadrillionths of a second.

"The neural network gave a first guess of the desired beam characteristics", Dr Scheinker recounts. "Then, adaptive feedback used it as a starting point to fine-tune, zoom in on, and track the time-varying optimal machine settings, based on real-time beam measurements. Although it focuses on just one immense and highly of this process are comparable to the operation of the human brain. While the first guess the neural network makes is not perfect, it makes its own decisions to find better alternatives and ultimately to reach the best possible solution without any compromises. For the LCLS FEL, the team's approach resulted in electron be automatically tuned to exactly match desired distributions. This was a first-of-its-kind step towards automatic tuning of FEL pulses towards creating distributions ideally suited for producing high-quality $X$-ray pulses.

\section{NEW EXPERIMENTA}

OPPORTUNITIES

Dr Scheinker's findings represent understanding of how machine learning can be used to optimise the performance of particle accelerators in real time. He also hopes that this active feedback control approach could be applied universally to any complex system which varies unpredictably over time, allowing each of their components to be simultaneously tuned during operation. For now, Dr Scheinker will work towards applying his adaptive neural networks to other aspects of particle accelerators beyond FELs. If his ideas cocome more widely adopted, they new research opportunities.

\section{Behind the Research}

\section{Dr Alexander Scheinker}

: ascheink@lanl.gov T: +1 5056958872

W: www.researchgate.net/profile/Alexander_Scheinker

Research Objectives

Dr Scheinker works on an adaptive machine learning approach that has the potential to optimise any complex time-varying system.

\section{Detail}

Los Alamos National Laboratory

PS BOx 1663

Los Alamos, NM 87544

Bio

Alexander Scheinker received a $\mathrm{PhD}$ in daptive control from UCSD and is a laboratory. He developed a new form of adaptive feedback for stabilisation
optimisation, control of unknown timevarying systems, and adaptive machine learning for time-varying systems
as particle accelerator beams.

as particle accelerator beams.

Funding
Los Alamos National Laboratory Laboratory Directed Research and Development (LDRD), Director's

References

- Scheinker, A., Bohler, D., Tomin, S., Kammering, R., Zagorodnov, I., Schlarb, H., Scholz, M., Beutne tuning for maximizing free electron laser puse energy Physical Review Accelerators and Beams, 22(8), 082802. Available at: https://doi.org/10.1103/ PhysRevAccelBeams.22.082802 Scheinker, A., Edelen, A., Bohler, D., Emma, C., and Lutman, A. (2018). Demonstration of modespace of electron beams in the Linac-coherent light source with Femtosecond resolution. Physical review letters, 121(4), 044801. Available at: https://doi. org/10.1103/PhysRevLett.121.044801 (2018). Constrained affine in control. International Journal of Robus and Nonlinear Control, 28(2), 568-581. Available at: https://doi.org//0.1002/rnc.3886 Scheinker, A., Scheinker, D. (2016). Bounded extremum seeking with discontinuous dithers. org/10.1016/jautomatica 2016.02023 for electron bunch profile prediction. Physical Review Special Topics-Accelerators and Beams, 18(10), PhysR PTAB. 18. 102801

\section{- Los Alamos}

Personal Response
What sparked your interest in neural networks?
II I became interested in neural networks because of their
ability to learn the underlying features of complex systems.
However, the majority of applications that I saw utilised
neural networks as static memorised maps for applications
such as image classification, surrogate modelling, or
anomaly detection. I was interested in dynamic, changing
systems for which we cannot memorise a set of rules.
I became interested in the idea of combining neural
networks with adaptive model-independent feedback. The
idea was that we could use the incredible power of neural
networks to learn an approximation of a complex global
landscape and use that as a map to guide a local model-
independent feedback that could zoom in on and crawl
around on the drifting changing landscape.
Which research areas would benefit from such
a feedback control system the most?
I think that any system in which the input-output relationships
change with time in an unpredictable way can benefit from
an adaptive machine learning approach. For example,
neural networks have proven to be extremely effective for
applications such as self-driving cars where pressing the
gas always makes the car speed up and pressing the brake
makes it slow down. However, in time-varying systems,
such as particle accelerators and their beams, increasing
the voltage of a magnet may increase the divergence of a
beam one moment and decrease it a few moments later,
depending on the beam itself and on other magnet settings.
For such systems, it is impossible to memorise anything
other than approximations of input-output relationships and
real-time data-based feedback must be used.

Collaborators

Alexander Scheinker has collaborated with his brother David Scheinker with his bronford University on general deplopment and proofs, as welthm European XFEL and the SLAC Nationa Accelerator Laboratory. 